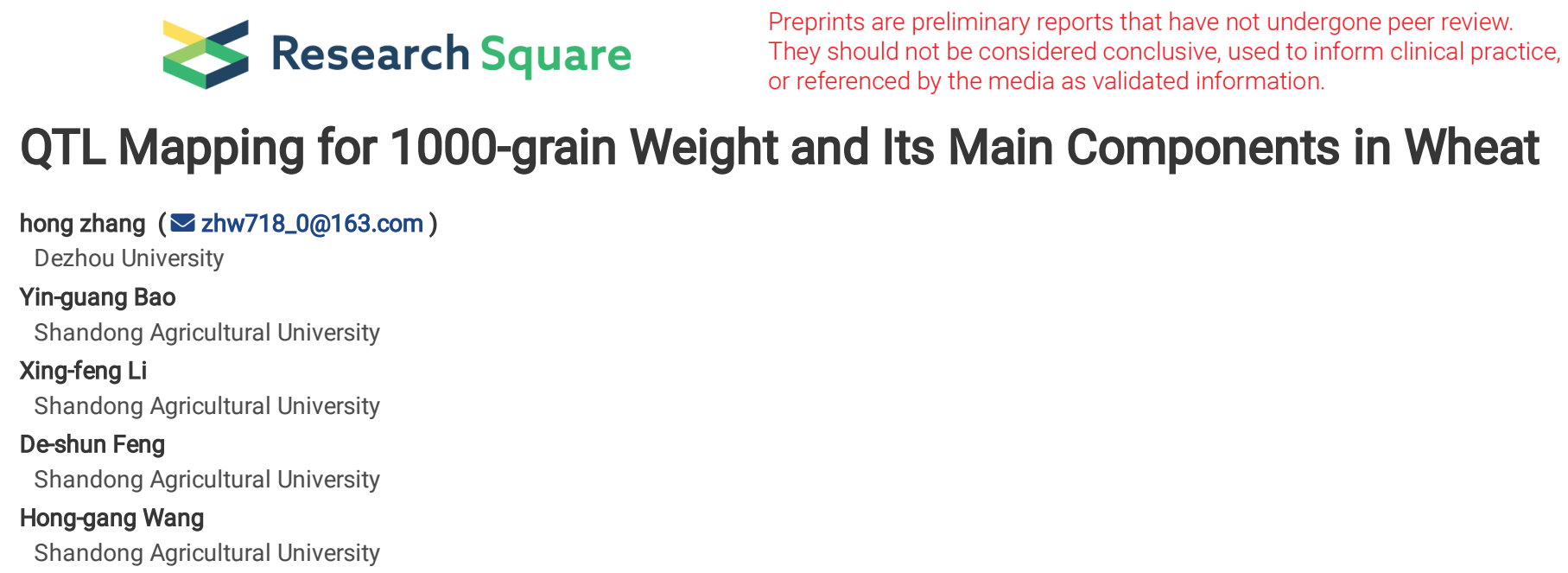

\section{QTL Mapping for 1000-grain Weight and Its Main Components in Wheat}




\section{Abstract}

In order to identify QTLs for 1000-grain weight and its main component traits in wheat, a high-density genetic link map was constructed using a $\mathrm{F}_{8: 9}$ recombined inbred line (RIL) population as material and exploiting the single nucleotide polymorphism (SNP) as well as PCR-based molecular markers, and to go a step further, QTL mapping for 1000-grain weight, grain length, and grain width in wheat was conducted. The linkage map was composed of 1257 loci formed by the 3916 markers, including 143 SSR markers and 3773 SNP markers, which were distributed on 22 chromosomes (2A chromosome formed a break point) with the total length of $2291.6 \mathrm{cM}$ and the average genetic distance between loci of $1.82 \mathrm{cM}$. A total of 41 qualitative trait loci (QTLs) on 19 chromosomes were detected, with contributions to phenotypic variance ranged from $3.59-58.49 \%$ for each QTL. Among these QTLs, two were detected in four environments, six in three environments, and 14 in two environments. Fifteen important loci with multi-effect were mapped on 9 chromosomes 1B, 2B, 3A, 4B, $5 A, 5 B, 6 A, 7 A$, and 7B, involving 37 QTLs that accounted for $90.2 \%$ of total numbers of QTLs detected. Of these 15 loci, including one on chromosome $7 A$ flanked by the markers Tdurum_contig77759_5 and BS00062425_51, which consisted of QTLs controlling TGW, GL, and GW and explained 4.2 to 58.49\% of the phenotypic variation in these traits. Thus, the chromosome intervals of the 15 loci were important areas controlling the expression of 1000 -grain weight and its main component traits in wheat.

\section{Introduction}

The grain length and grain width are two important factors that influence wheat 1000-grain weight. Between these two factors, grain width matters more. The 1000-grain weight, grain length and grain width of wheat are all quantitative traits controlled by multiple genes (Cui et al. 2011; Ramya et al. 2010). Yu Manli et al. (2014) detected six QTLs controlling 1000-grain weight Located on 1D, 2B, 3D, 6D and 7A chromosomes, 12 QTLs controlling grain length on 5A, 7A, 1B, 2B, 3B, 1D, 6D and 7D chromosomes, and four QTLs controlling grain width on 1B, 2B and 6D chromosomes. Golabadi et al. (2010) identified 10 SSR markers tight interlocked with TGW respectively on chromosomes $1 \mathrm{~A}, 5 \mathrm{~A}, 6 \mathrm{~A}, 3 \mathrm{~B}$ and 7B by Single marker analysis. Using a RIL population containing 185 families,Ramya et al. (2010) detected 10 QTLs controlling 1000-grain weight located on 1A, 1D, 2B, 2D, 4B, 5B and 6B chromosomes, six QTLs controlling grain length on chromosomes 5B and 5D, and nine QTLs controlling grain width on chromosomes 1D, 2B, 2D, 4B, 5B and 5D.

Although QTL mapping for 1000-grain weight and its component traits has already been reported early these years, there are great differences in QTLs detection applying different genetic materials. In addition, the accuracy of localization also varies greatly due to different types of molecular markers and the different densities of the genetic maps constructed; therefore, it is necessary to use different experimental materials and various types of molecular markers to construct high-density maps for all-round research. In this study, a high-density genetic link map was constructed using a $\mathrm{F}_{8: 9}$ recombined inbred line (RIL) population as material and exploiting the SNP and PCR-based molecular markers, and QTL mapping for 1000-grain weight, grain length and grain width in wheat was conducted in order to reveal the relationships at molecular level among 1000-grain weight, grain length and grain width.

\section{Materials And Methods}

Test material

The RIL population was constructed by crossing Weimai 8 as female parent and Ji 8901 as male parent, containing 163 lines and the WJ Group for short. Among them, Wei Mai 8 was a 1BL/1RS translocation line, Ji 8901 contained normal 1B chromosome. Weimai 8 had larger grain, whose 1000-grain weight was about 48.7g, while Ji 8901 had smaller grain, with a $39.5 \mathrm{~g} 1000$-grain-weight.

Field trials

A test point was set in Tai'an $\left(E_{1}\right)$ in 2013. Three test points were set in Tai'an $\left(E_{2}\right)$, Zaozhuang $\left(E_{3}\right)$ and Jining $\left(E_{4}\right)$ in 2014 , respectively. The experiment was designed with random block design, ordinal arrangement, and interval contrast design with 3 biological repeats. The plant sown with the single seed was planted two lines each time, with the row $(120 \mathrm{~cm} * 23.5 \mathrm{~cm}$, length *width). The plant distance was $10 \mathrm{~cm}$. At the mature stage, 5 plants were collected from the middle of the row, and 1000-grain weight, grain length and grain width were measured by SC-G automatic seed testing analysis and 1000-grain weight meter.

DNA extraction and molecular marker polymorphism detection

The total DNA of plant was obtained by SDS method. The materials were washed with $70 \%$ ethanol $2-3$ times, dried naturally, and then dissolved in $100 \mu \mathrm{L} 1 \times$ TE. The concentration was determined by Thermo Nanodrop 2000 and the final concentration of DNA was adjusted to 100ng/uL. DNA powder was prepared by vacuum extraction at low temperature and sent to Illumina company of the United States for genome scanning using Infinium ISELECT 90K SNP chip. Polymorphism analysis was performed using Genome studio V1.0 software. At the same time, 2408 pairs of PCR-based molecular markers including G-SSR, EST-SSR, STS and ISSR were used to detect the polymorphism of parents and population (Zhang 2013).

Genetic mapping and QTL analysis

Excel and SPSS13.0 were used for data collation and statistical analysis. JoinMap4.0 was used to construct the linkage map, and Kosambi was used as the map distance function. The parameters were set as: REC $=0.35, \mathrm{LOD}=4.0$ and JUMP $=5$. IciMapping v3.2 (http://www.isbreeding.net/) was applied to QTL analysis of phenotypic value, and LOD critical value was 2.5, step value was 1. Map chart 2.1 (Voorrips 2002) was used for QTL labeling. The average value of three replicates was used as input data, and the QTL was named by Q + Abbreviation of character name + Chromosome number (Mclntosh et al. 2007).

\section{Results}

Page 2/11 
A total of 81587 SNP markers were used to detect the population, among which 12613 markers were polymorphic, and 3811 markers with good polymorphism were selected by removing more than $25 \%$ of the sites with partial segregation or deletion. The 675 pairs of polymorphic primers were detected in parents Weimai 8 and JI 8901 among 2408 pairs of PCR-based molecular markers. The distinct and different loci in RIL population were amplified by 183 pairs of primers and 201 differential loci were formed. The primary frame map of wheat genetic linkage was constructed by PCR-based molecular markers, and then encrypted by SNP markers.

The linkage map was composed of 3916 markers, including 143 SSR markers and 3773 SNP markers, forming 1257 loci, which were distributed on 22 chromosomes (2A chromosome formed a break point), and the total length was $2291.6 \mathrm{cM}$. The average genetic distance between loci was $1.82 \mathrm{cM}$. The number of single chromosome marker sites was from 3 to 525 . Among them, gene group A contains 541 sites, whose total length is $925.9 \mathrm{cM}$ and the average genetic distance between sites is $1.71 \mathrm{cM}$; while gene group B contains 574 sites, whose total length is $899.1 \mathrm{cM}$ and the average genetic distance between sites is $1.57 \mathrm{cM}$. The Loci number of gene group D was the smallest, containing 142 Loci. The total length was $466.6 \mathrm{cM}$, and the average genetic distance between loci was $3.29 \mathrm{Cm}$ (Zhang 2013).

Analysis of phenotypic data

Significant differences were detected in the 1000-grain weight, grain length and grain width of Weimai 8 and Ji 8901 under four environments, suggesting that there were significant differences in 1000-grain weight and its main component traits between the two parents. Within the population, the absolute values of Kurtosis and skewness were all less than 1 (Table 1), which was consistent with the genetic characteristics of quantitative traits controlled by micro-effective polygenes. The correlation analysis of characters showed that the average correlation coefficients of 1000-grain weight under four environments, and the grain length was 0.46 while and grain width was 0.75 , respectively. The average correlation coefficients of grain length and grain width were 0.29 . In addition, the estimated broad sense heritability of 1000 -grain weight, grain length and grain width were $0.85,0.77$ and 0.86 , respectively.

Table 1 The variation of phenotypic traits in WJ population under different environments

\begin{tabular}{|c|c|c|c|c|c|c|c|c|c|}
\hline \multirow{2}{*}{$\begin{array}{l}\text { Traits } \\
(\mathrm{h} \%)^{a}\end{array}$} & \multirow[t]{2}{*}{ Environment ${ }^{b}$} & \multicolumn{2}{|c|}{ Parental mean } & \multicolumn{6}{|c|}{ WJ RIL Population } \\
\hline & & Weimai $8^{c}$ & Ji $8901^{c}$ & Minimum & Maximum & Mean & $S_{t d}{ }^{d}$ & Skewness & Kurtosis \\
\hline \multirow{4}{*}{$\begin{array}{l}\text { TGW (g) } \\
0.85\end{array}$} & $\mathrm{E}_{1}$ & $52.27 \mathrm{aA}$ & $32.51 \mathrm{bB}$ & 30.58 & 57.29 & 45.35 & 4.85 & -0.42 & 0.83 \\
\hline & $\mathrm{E}_{2}$ & $43.55 \mathrm{aA}$ & $31.60 \mathrm{bB}$ & 25.13 & 54.70 & 41.48 & 5.91 & -0.34 & -0.29 \\
\hline & $E_{3}$ & 37.83 aA & $34.00 \mathrm{bA}$ & 24.42 & 47.55 & 34.86 & 5.35 & 0.24 & -0.64 \\
\hline & $\mathrm{E}_{4}$ & $52.96 \mathrm{aA}$ & $36.10 \mathrm{bB}$ & 27.54 & 55.32 & 42.23 & 4.91 & -0.25 & 0.46 \\
\hline \multirow{4}{*}{$\begin{array}{l}\mathrm{GL}(\mathrm{mm}) \\
0.77\end{array}$} & $\mathrm{E}_{1}$ & $3.70 \mathrm{aA}$ & $3.28 \mathrm{bB}$ & 2.89 & 3.78 & 3.27 & 0.19 & 0.08 & -0.50 \\
\hline & $\mathrm{E}_{2}$ & $3.35 \mathrm{aA}$ & $3.12 \mathrm{bA}$ & 2.98 & 4.00 & 3.40 & 0.21 & 0.25 & -0.37 \\
\hline & $E_{3}$ & $3.35 \mathrm{aA}$ & $3.03 \mathrm{bB}$ & 3.06 & 3.93 & 3.47 & 0.21 & 0.11 & -0.79 \\
\hline & $\mathrm{E}_{4}$ & $3.48 \mathrm{aA}$ & $3.19 \mathrm{bA}$ & 3.03 & 4.30 & 3.55 & 0.21 & 0.44 & 0.34 \\
\hline \multirow{4}{*}{$\begin{array}{l}\text { GW }(\mathrm{mm}) \\
0.86\end{array}$} & $\mathrm{E}_{1}$ & $1.94 \mathrm{aA}$ & 1.64 bB & 1.38 & 1.94 & 1.67 & 0.09 & -0.50 & 0.84 \\
\hline & $\mathrm{E}_{2}$ & $1.80 \mathrm{aA}$ & $1.50 \mathrm{bB}$ & 1.40 & 1.89 & 1.65 & 0.09 & -0.13 & -0.12 \\
\hline & $\mathrm{E}_{3}$ & $1.60 \mathrm{aA}$ & 1.48 bA & 1.42 & 1.85 & 1.61 & 0.08 & 0.17 & -0.25 \\
\hline & $\mathrm{E}_{4}$ & $1.79 \mathrm{aA}$ & $1.60 \mathrm{bB}$ & 1.55 & 1.95 & 1.76 & 0.09 & -0.16 & -0.66 \\
\hline \multicolumn{10}{|c|}{ a Arabic numerals in parentheses are estimated broad-sense heritability of the corresponding traits } \\
\hline \multicolumn{10}{|c|}{${ }^{b} E_{1}, E_{2}, E_{3}, E_{4}$ means 2013 in Tai'an, 2014 in Tai'an, in Zaozhuang, and in Jining, respectively } \\
\hline \multicolumn{10}{|c|}{$\begin{array}{l}\text { C Small letters 'a' and ' } b \text { ' means significance of difference when } P<0.05 \text {, and capital letters ' } A \text { ' and 'B' means significance of difference when } P<0.01 \\
\text { between two parents. }\end{array}$} \\
\hline \multicolumn{10}{|c|}{ d Std Standard deviation } \\
\hline
\end{tabular}

QTL analysis

\section{QTLs concerned with 1000-grain weight}


17 QTLs concerned with 1000-grain weight were detected on chromosomes 1A, 1B, 2B, 3A, 3B, 4B, 5A, 5B, 5D, 6A, 7A, and 7B which accounted for $4.20 \%-23.96 \%$ of the phenotypic variation, respectively. Nine of them were main-effect QTLs individually accounting for $>10 \%$ of the phenotypic variation. QGWT-5B was a main-effect QTL with positive additive effect, derived from Weimai 8, it was simultaneously detected in four environments, and accounted for $4.63 \%-10 \%$ of the phenotypic variation in different environments. Three QTLs were simultaneously detected in three environments. Among them, QGWT-1B.1 and QGWT-2B.2 showed the negative additive effects derived from Ji 8901, accounting for $9.86 \%-16.92 \%$ and $4.48 \%-10.21 \%$ of phenotypic variation in different environments, respectively, and QGWT-4B.1 showed the positive additive effects derived from Weimai 8, accounting for $5.72 \%-16.57 \%$ of phenotypic variation in different environments. Five QTLs QGWT-1B.2, QGWT-2B.1, QGWT-4B.2, QGWT-5A.1 and QGWT-5A.2 were simultaneously detected in two environments, all with positive additive effects derived from Weimai 8. Among them, QGWT-4B. 2 accounted for $23.96 \%$ of phenotypic variation in E2 environment, QGWT-5A.2 accounted for $17.05 \%$ and $13.47 \%$ of phenotypic variation in $\mathrm{E}_{2}$ and $\mathrm{E}_{4}$, respectively.

\section{QTLs concerned with grain length}

A total of 13 additive effect QTLs concerned with grain length were detected on chromosomes 1A, 1B, 1D, 2B, 3B, 4A, 5A, 5B, 7A, and 7B, and accounted for $3.59 \%-58.49 \%$ of phenotypic variation. Four of them were main-effect QTLs individually accounting for $>10 \%$ of the phenotypic variation. $Q G L-1 D$ was a major QTL simultaneously detected in 4 environments, with positive additive effect derived from Weimai 8 , and accounting for $9.46 \%-27.52 \%$ of the phenotypic variation in various environments. Two QTLs $Q G L-1 B$ and $Q G L-2 B$ were simultaneously detected in three environments, both with the positive additive effects derived from Weimai 8, and accounting for < 10\% of the phenotypic variation. Five QTLs QGL-1A, QGL-3B.1, QGL-3B.2, QGL-7A.1 and QGL-7A.2 were simultaneously detected in two environments. Among them, only QGL-7A.2 additive effect derived from Weimai 8, the contribution rate was $58.49 \%$ in $\mathrm{E}_{1}$ environment. Five QTLs were only detected in a single environment. QGL-5B was a main-effect QTL and accounted for $12.57 \%$ of the phenotypic variation.

\section{QTLs concerned with grain width}

A total of 21 additive effect QTLs concerned with grain width were detected on chromosomes 1A, 2A, 2B, 3A, 4A, 4B, 4D, 5A, 5B, 6A, 6B, 7A and 7B, and they accounted for $4.32 \%-20.62 \%$ of phenotypic variation. Nine of them were main-effect QTLs individually accounting for $>10 \%$ of the phenotypic variation. $Q G W$ $7 A$ was a major QTL simultaneously detected in 3 environments, with negative additive effect derived from Ji 8901 , and it accounts for $9.6 \%-15.5 \%$ of the phenotypic variation in different environments. Besides, four QTLs QGW-2A2, QGW-4D.1, QGW-5A.3, and QGW-1B were simultaneously detected in two environments, but only $Q G W-5 A$.3 has the negative additive effects derived from Ji 8901 and it accounts for $<10 \%$ of the phenotypic variation. $Q G W-2 A 2$ and QGW-1B were the main-effect QTLs. 16 QTLs were detected only in one single environment and six of them were the main-effect QTLs.

\section{Loci with multiple effects}

As is shown above, some QTLs for 1000-grain weight, grain length and grain width were located in the same chromosome segment and belonged to QTLs with multiple effects by comprehensive analysis. A total of 15 loci with multi-effect were identified (Table 3). It's well-worth mentioning that one of them was located on chromosome 7A, which simultaneously controlled 1000-grain weight, grain length and grain width. Eleven loci with multiple-effects simultaneously controlled 1000-grain weight as well as grain width, and they were located on chromosomes 1B, 2B, 3A, 4B, 5A, 6A and 7B, respectively. What's more, two QTLs with multiple-effects simultaneously controlled 1000-grain weight and grain length, located on chromosomes 1B and 5B, respectively. One QTL with multipleeffect simultaneously controlled grain length and grain width, located on chromosomes 5B. (Fig. 1, Table 2). 
Table 2

QTLs for 1000-grain weight and its main components in WJ population

\begin{tabular}{|c|c|c|c|c|c|c|c|c|}
\hline Trait & QTL & Environment & $\begin{array}{l}\text { Chr. } \\
\text { a }\end{array}$ & Left Marker ${ }^{b}$ & Right Marker & LOD & PVE (\%) ${ }^{c}$ & $A_{1}$ \\
\hline \multirow[t]{17}{*}{$G W T$} & $\begin{array}{l}\text { QGWT } \\
1 A\end{array}$ & $E_{2}$ & $1 A$ & CFE26.2 & CWM102 & 3.43 & 5.95 & -1 \\
\hline & $\begin{array}{l}\text { QGWT } \\
1 B .1\end{array}$ & $\mathrm{E}_{1} / \mathrm{E}_{2} / \mathrm{E}_{3}$ & 1B & CAP11_c7969_315 & BS00084990_51 & 6.18/4.34./6.71 & $16.92 / 14.4 / 9.86$ & -3 \\
\hline & $\begin{array}{l}\text { QGWT } \\
1 B .2\end{array}$ & $E_{1} / E_{2}$ & 1B & BS00021934_51 & wsnp_Ex_c6611_114520 & $5.43 / 3.74$ & $8.93 / 9.8$ & 1. \\
\hline & $\begin{array}{l}\text { QGWT } \\
2 B .1\end{array}$ & $\mathrm{E}_{4} / \mathrm{E}_{3}$ & $2 B$ & wsnp_Ex_c1962_369626 & Xgwm148 & $4.26 / 6.37$ & $8.32 / 9.53$ & 0. \\
\hline & $\begin{array}{l}\text { QGWT } \\
2 B .2\end{array}$ & $\mathrm{E}_{4} / \mathrm{E}_{3} / \mathrm{E}_{2}$ & $2 B$ & Xcinau175.2 & Xcinau175.1 & $6.25 / 3.05 / 3.79$ & $10.21 / 4.48 / 7.24$ & -1 \\
\hline & $\begin{array}{l}\text { QGWT } \\
3 A\end{array}$ & $\mathrm{E}_{4}$ & $3 A$ & GENE-1549_110 & CAP7_c9218_89 & 6.31 & 11.26 & -0 \\
\hline & $\begin{array}{l}Q G W T- \\
3 B\end{array}$ & $E_{3}$ & $3 B$ & GENE-1662_108 & wsnp_CAP11_c59_99317 & 4.74 & 6.72 & 1. \\
\hline & $\begin{array}{l}\text { QGWT } \\
4 B .1\end{array}$ & $\mathrm{E}_{1} / \mathrm{E}_{2} / \mathrm{E}_{4}$ & $4 \mathrm{~B}$ & wsnp_Ex_c3119_5763762 & RAC875_c19303_228 & $3.34 / 5.67 / 5.63$ & $5.72 / 16.57 / 9.41$ & 1. \\
\hline & $\begin{array}{l}\text { QGWT } \\
4 B .2\end{array}$ & $\mathrm{E}_{2} / \mathrm{E}_{4}$ & $4 \mathrm{~B}$ & Ex_c7059_1198 & Kukri_c35140_75 & $6.57 / 3.13$ & $23.96 / 4.85$ & 4. \\
\hline & $\begin{array}{l}\text { QGWT } \\
5 A .1\end{array}$ & $\mathrm{E}_{1} / \mathrm{E}_{3}$ & $5 \mathrm{~A}$ & BS00094095_51 & wsnp_CAP7_c2282_1107 & $4.41 / 2.94$ & $7.15 / 4.65$ & 1. \\
\hline & $\begin{array}{l}\text { QGWT } \\
5 A .2\end{array}$ & $\mathrm{E}_{2} / \mathrm{E}_{4}$ & $5 \mathrm{~A}$ & Excalibur_c51589_200 & Excalibur_c7556_381 & $8.75 / 7.78$ & $17.05 / 13.47$ & 2. \\
\hline & $\begin{array}{l}\text { QGWT } \\
5 A .3\end{array}$ & $\mathrm{E}_{4}$ & $5 \mathrm{~A}$ & Excalibur_c52167_355 & Xgwm156 & 6.76 & 11.44 & -1 \\
\hline & $\begin{array}{l}Q G W T- \\
5 B\end{array}$ & $\mathrm{E}_{1} / \mathrm{E}_{2} / \mathrm{E}_{3} / \mathrm{E}_{4}$ & $5 B$ & BobWhite_rep_c49058 & IACX7928 & $2.92 / 4.53 / 5.96 / 4.12$ & $10 / 4.63 / 12.8 / 8.37$ & 2. \\
\hline & $\begin{array}{l}Q G W T- \\
5 D\end{array}$ & $E_{3}$ & $5 \mathrm{D}$ & Xgpw5207 & $X g d m 3$ & 3.92 & 10.17 & -2 \\
\hline & $\begin{array}{l}Q G W T- \\
6 A\end{array}$ & $E_{2}$ & $6 \mathrm{~A}$ & wsnp_Ex_rep_c68165_6 & BS00034886_51 & 4.52 & 7.53 & -1 \\
\hline & $\begin{array}{l}Q G W T- \\
7 A\end{array}$ & $E_{3}$ & $7 \mathrm{~A}$ & Tdurum_contig77759_5 & BS00062425_51 & 3.09 & 4.2 & -1 \\
\hline & $\begin{array}{l}Q G W T- \\
7 B\end{array}$ & $\mathrm{E}_{4}$ & 7B & BARC014 & BobWhite_c12256_96 & 2.84 & 4.22 & 0. \\
\hline \multirow[t]{7}{*}{$\mathrm{GL}$} & $\begin{array}{l}\text { QGL- } \\
1 A\end{array}$ & $\mathrm{E}_{3} / \mathrm{E}_{4}$ & $1 \mathrm{~A}$ & Xedm24wsnp_ & Ex_c48407_53323 & $4.53 / 4.05$ & $5.76 / 5.77$ & 0. \\
\hline & $\begin{array}{l}Q G L- \\
1 B\end{array}$ & $\mathrm{E}_{2} / \mathrm{E}_{3} / \mathrm{E}_{4}$ & 1B & BS00077498_51 & wsnp_Ex_c6611_114519 & $4 / 5.11 / 4.8$ & $9.63 / 9.66 / 6.63$ & 0. \\
\hline & $\begin{array}{l}Q G L- \\
1 D\end{array}$ & $\mathrm{E}_{1} / \mathrm{E}_{2} / \mathrm{E}_{3} / \mathrm{E}_{4}$ & $1 \mathrm{D}$ & Tdurum_contig28703_2 & Kukri_c14149_462 & 17.29/7.21/13.98/7.14 & $27.52 / 11.89 / 17.42 / 9.46$ & 0. \\
\hline & $\begin{array}{l}Q G L- \\
2 B\end{array}$ & $\mathrm{E}_{2} / \mathrm{E}_{3} / \mathrm{E}_{4}$ & $2 B$ & Ra_c5004_1902 & BobWhite_c13295_407 & $3.43 / 5.57 / 3.63$ & $4.36 / 6.49 / 4.99$ & 0. \\
\hline & $\begin{array}{l}\text { QGL- } \\
3 B .1\end{array}$ & $E_{2} / E_{3}$ & $3 B$ & RAC875_c15109_510 & BS00048659_51 & $6.11 / 8.88$ & $8.01 / 11.12$ & 0. \\
\hline & $\begin{array}{l}\text { QGL- } \\
3 B .2\end{array}$ & $\mathrm{E}_{3} / \mathrm{E}_{4}$ & 3B & BS00046052_51 & wsnp_CAP11_c323_2638 & $4.08 / 7.45$ & $5.65 / 9.3$ & 0. \\
\hline & $\begin{array}{l}Q G L- \\
4 A\end{array}$ & $E_{3}$ & $4 \mathrm{~A}$ & BARC070.2 & Jagger_c4331_105 & 3.34 & 3.59 & 0. \\
\hline
\end{tabular}

a Chromosome on which the QTL was detected

${ }^{\mathrm{b}}$ Flanking marker

${ }^{\mathrm{c}}$ Percentage phenotypic variance explained by additive effects

${ }^{d}$ Estimate of the additive effect of the QTL. Positive values indicate that Weimai 8 alleles increase the trait value; negative values indicate that Weimai 8 allel value 


\begin{tabular}{|c|c|c|c|c|c|c|c|c|}
\hline \multirow[t]{7}{*}{ Trait } & QTL & Environment & $\begin{array}{l}\text { Chr. } \\
\text { a }\end{array}$ & Left Marker $^{b}$ & Right Marker & LOD & PVE (\%) ${ }^{c}$ & $A_{1}$ \\
\hline & $\begin{array}{l}\text { QGL- } \\
5 A\end{array}$ & $\mathrm{E}_{2}$ & $5 \mathrm{~A}$ & BS00090309_51 & BS00083507_51 & 4.17 & 4.57 & 0. \\
\hline & $\begin{array}{l}Q G L- \\
5 B .1\end{array}$ & $\mathrm{E}_{1}$ & $5 B$ & Xmag1242 & BobWhite_c28958_178 & 8.26 & 12.57 & -0 \\
\hline & $\begin{array}{l}Q G L- \\
5 B .2\end{array}$ & $\mathrm{E}_{3}$ & $5 B$ & BobWhite_rep_c49058 & IACX7928 & 3.3 & 4.6 & 0. \\
\hline & $\begin{array}{l}Q G L- \\
7 A .1\end{array}$ & $E_{2} / E_{3}$ & $7 \mathrm{~A}$ & RAC875_c21165_1058 & RFL_Contig2834_890 & $3.19 / 3.84$ & $8.32 / 8.25$ & 0. \\
\hline & $\begin{array}{l}\text { QGL- } \\
7 A .2\end{array}$ & $\mathrm{E}_{1} / \mathrm{E}_{3}$ & $7 \mathrm{~A}$ & Tdurum_contig77759_5 & BS00062425_51 & $15.16 / 2.66$ & $58.49 / 5.18$ & -1 \\
\hline & $\begin{array}{l}Q G L- \\
7 B\end{array}$ & $\mathrm{E}_{2}$ & 7B & Xgwm537 & Kukri_c48870_115 & 2.52 & 6.42 & -0 \\
\hline \multirow[t]{18}{*}{$\mathrm{GW}$} & $\begin{array}{l}Q G W- \\
1 B\end{array}$ & $\mathrm{E}_{2}$ & 1B & CAP11_c7969_315 & BS00084990_51 & 4.74 & 7.94 & -0 \\
\hline & $\begin{array}{l}Q G W- \\
2 A_{2}\end{array}$ & $\mathrm{E}_{2} / \mathrm{E}_{3}$ & $2 \mathrm{~A}_{2}$ & RAC875_c38018_278 & IAAV6070 & $2.61 / 4.54$ & $5.4 / 12.94$ & 0. \\
\hline & $\begin{array}{l}Q G W- \\
2 B .1\end{array}$ & $\mathrm{E}_{3}$ & $2 B$ & wsnp_Ex_c1962_369626 & Xgwm148 & 8.5 & 19.22 & 0. \\
\hline & $\begin{array}{l}Q G W- \\
2 B .2\end{array}$ & $E_{2}$ & $2 \mathrm{~B}$ & Xcinau175.2 & Xcinau175.1 & 4.39 & 7.6 & -0 \\
\hline & $\begin{array}{l}Q G W- \\
3 A\end{array}$ & $\mathrm{E}_{4}$ & $3 A$ & GENE-1549_110 & CAP7_c9218_89 & 3.91 & 7.53 & -0 \\
\hline & $\begin{array}{l}Q G W- \\
4 A\end{array}$ & $\mathrm{E}_{2}$ & $4 \mathrm{~A}$ & wsnp_Ex_c5690_999430 & Kukri_c6221_742 & 2.96 & 4.58 & -0 \\
\hline & $\begin{array}{l}Q G W- \\
4 B .1\end{array}$ & $\mathrm{E}_{2}$ & $4 \mathrm{~B}$ & wsnp_Ex_c3119_5763762 & RAC875_C19303_228 & 5.61 & 15.86 & 0. \\
\hline & $\begin{array}{l}Q G W- \\
4 B .2\end{array}$ & $\mathrm{E}_{2}$ & $4 \mathrm{~B}$ & Ex_c7059_1198 & Kukri_c35140_75 & 9.95 & 20.62 & 0. \\
\hline & $\begin{array}{l}\text { QGW- } \\
4 B .3\end{array}$ & $\mathrm{E}_{1}$ & $4 \mathrm{~B}$ & Xgwm251.1 & RAC875_c27536_611 & 4.34 & 5.3 & 0. \\
\hline & $\begin{array}{l}Q G W- \\
4 B .4\end{array}$ & $\mathrm{E}_{4}$ & $4 \mathrm{~B}$ & Tdurum_contig60051_1 & Tdurum_contig50861_5 & 7.15 & 14.76 & 0. \\
\hline & $\begin{array}{l}Q G W- \\
4 D\end{array}$ & $\mathrm{E}_{1} / \mathrm{E}_{2}$ & $4 \mathrm{D}$ & wsnp_Ex_rep_c67296_6 & Kukri_c56182_129 & $3.68 / 2.97$ & $4.32 / 7.46$ & 0. \\
\hline & $\begin{array}{l}Q G W- \\
5 A .1\end{array}$ & $\mathrm{E}_{4}$ & $5 \mathrm{~A}$ & BS00094095_51 & wsnp_CAP7_c2282_1107 & 4.96 & 9.13 & 0. \\
\hline & $\begin{array}{l}\text { QGW- } \\
5 A .2\end{array}$ & $E_{3}$ & $5 \mathrm{~A}$ & Excalibur_c51589_200 & Excalibur_c7556_381 & 6.31 & 16.6 & 0. \\
\hline & $\begin{array}{l}\text { QGW- } \\
5 A .3\end{array}$ & $\mathrm{E}_{3} / \mathrm{E}_{4}$ & $5 \mathrm{~A}$ & Excalibur_c52167_355 & Xgwm156 & $2.81 / 4.01$ & $6.39 / 7.34$ & -0 \\
\hline & $\begin{array}{l}\text { QGW- } \\
5 B .1\end{array}$ & $E_{3}$ & $5 B$ & BS00009335_51 & TA012745-0706 & 6.5 & 13.39 & -0 \\
\hline & $\begin{array}{l}Q G W- \\
5 B .2\end{array}$ & $\mathrm{E}_{1}$ & $5 B$ & Xmag1242 & BobWhite_c28958_178 & 7.3 & 9.37 & -0 \\
\hline & $\begin{array}{l}Q G W- \\
6 A\end{array}$ & $\mathrm{E}_{4}$ & $6 \mathrm{~A}$ & wsnp_Ex_rep_c68165_6 & BS00034886_51 & 3.68 & 6.91 & -0 \\
\hline & $\begin{array}{l}Q G W- \\
6 B .1\end{array}$ & $\mathrm{E}_{4}$ & $6 B$ & BS00027942_51 & Xwmc494 & 4.02 & 8.2 & -0 \\
\hline
\end{tabular}

a Chromosome on which the QTL was detected

${ }^{\mathrm{b}}$ Flanking marker

${ }^{\mathrm{c}}$ Percentage phenotypic variance explained by additive effects

${ }^{d}$ Estimate of the additive effect of the QTL. Positive values indicate that Weimai 8 alleles increase the trait value; negative values indicate that Weimai 8 allel value 


\begin{tabular}{|c|c|c|c|c|c|c|c|c|}
\hline Trait & QTL & Environment & $\begin{array}{l}\text { Chr. } \\
\text { a }\end{array}$ & Left Marker $^{b}$ & Right Marker & LOD & $\operatorname{PVE}(\%)^{c}$ & $A_{1}$ \\
\hline & $\begin{array}{l}\text { QGW- } \\
6 B .2\end{array}$ & $\mathrm{E}_{4}$ & $6 \mathrm{~B}$ & TA003403-0617 & GENE-4208_229 & 4.29 & 9.55 & -0 \\
\hline & $\begin{array}{l}\text { QGW- } \\
7 A\end{array}$ & $\mathrm{E}_{2} / \mathrm{E}_{3} / \mathrm{E}_{4}$ & $7 \mathrm{~A}$ & Tdurum_contig77759_5 & BS00062425_51 & $3.84 / 4.78 / 4.49$ & $10.34 / 9.6 / 15.5$ & -0 \\
\hline & $\begin{array}{l}Q G W- \\
7 B\end{array}$ & $\mathrm{E}_{1} / \mathrm{E}_{4}$ & 7B & BARC014 & BobWhite_c12256_96 & $3.32 / 7.34$ & $12.43 / 15.57$ & 0. \\
\hline
\end{tabular}

a Chromosome on which the QTL was detected

${ }^{\mathrm{b}}$ Flanking marker

${ }^{\mathrm{c}}$ Percentage phenotypic variance explained by additive effects

${ }^{d}$ Estimate of the additive effect of the QTL. Positive values indicate that Weimai 8 alleles increase the trait value; negative values indicate that Weimai 8 allel value

Table 3

Loci with multiple effects detected

\begin{tabular}{|c|c|c|c|c|c|c|}
\hline Trait ${ }^{a}$ & $\mathrm{QTL}^{\mathrm{b}}$ & Env. ${ }^{c}$ & Chr. & Left Marker & Right Marker & PVE (\%) ${ }^{d}$ \\
\hline $\begin{array}{l}\text { GWT, GL, } \\
\text { GW }\end{array}$ & $\begin{array}{l}\text { QGWT-7A, QGL-7A.2, QGW- } \\
7 A\end{array}$ & $\begin{array}{l}E_{3,} E_{1} / E_{3} \\
E_{2} / E_{3} / E_{4}\end{array}$ & $7 A$ & Tdurum_contig77759_5 & BS00062425_51 & $\begin{array}{l}4.2,58.49 / 5.18 \\
10.34 / 9.6 / 15.5\end{array}$ \\
\hline \multirow[t]{2}{*}{ GWT, GL } & QGWT-1B.2, QGL-1B & $\mathrm{E}_{1} / \mathrm{E}_{2}, \mathrm{E}_{2} / \mathrm{E}_{3} / \mathrm{E}_{4}$ & $1 \mathrm{~B}$ & BS00021934_51 & wsnp_Ex_c6611_114520 & $\begin{array}{l}\text { 8.93/9.8, } \\
9.63 / 9.66 / 6.63\end{array}$ \\
\hline & QGWT-5B, QGL-5B.2 & $\mathrm{E}_{1} / \mathrm{E}_{2} / \mathrm{E}_{3} / \mathrm{E}_{4}, \mathrm{E}_{3}$ & $5 B$ & Kukri_c45713_151 & wsnp_Ex_rep_c66667_6 & 10/4.63/12.8/8.37, 4.6 \\
\hline \multirow[t]{11}{*}{ GWT, GW } & QGWT-1B.1, QGW-1B & $\mathrm{E}_{1} / \mathrm{E}_{2} / \mathrm{E}_{3}, \mathrm{E}_{2}$ & 1B & CAP11_C7969_315 & BS00084990_51 & $16.92 / 14.4 / 9.86,7.94$ \\
\hline & QGWT-2B.1, QGW-2B.1 & $E_{4} / E_{3}, E_{3}$ & $2 \mathrm{~B}$ & wsnp_Ex_c1962_369626 & Xgwm148 & $8.32 / 9.53,19.22$ \\
\hline & QGWT-2B.2, QGW-2B.2 & $\mathrm{E}_{4} / \mathrm{E}_{3} / \mathrm{E}_{2}, \mathrm{E}_{2}$ & $2 \mathrm{~B}$ & Xcinau175.2 & Xcinau 175.1 & $10.21 / 4.48 / 7.24,7.6$ \\
\hline & QGWT-3A, QGW-3A & $\mathrm{E}_{4}, \mathrm{E}_{4}$ & $3 A$ & GENE-1549_110 & CAP7_C9218_89 & $11.26,7.53$ \\
\hline & QGWT-4B.1, QGW-4B.1 & $\mathrm{E}_{1} / \mathrm{E}_{2} / \mathrm{E}_{4}, \mathrm{E}_{2}$ & $4 \mathrm{~B}$ & wsnp_Ex_c3119_5763762 & RAC875_c19303_228 & $5.72 / 16.57 / 9.41,15.86$ \\
\hline & $Q G W T-4 B .2, Q G W-4 B .2$ & $E_{2} / E_{4}, E_{2}$ & $4 \mathrm{~B}$ & $E x_{-} c 7059 \_1198$ & Kukri_c35140_75 & $23.96 / 4.85,20.62$ \\
\hline & QGWT-5A.1, QGW-5A.1 & $E_{1} / E_{3}, E_{4}$ & $5 \mathrm{~A}$ & BS00094095_51 & wsnp_CAP7_c2282_1107 & $7.15 / 4.65,9.13$ \\
\hline & QGWT-5A.2, QGW-5A.2 & $E_{2} / E_{4}, E_{3}$ & $5 \mathrm{~A}$ & Excalibur_c51589_200 & Excalibur_c7556_381 & $17.05 / 13.47,16.6$ \\
\hline & QGWT-5A.3, QGW-5A.3 & $\mathrm{E}_{4}, \mathrm{E}_{3} / \mathrm{E}_{4}$ & $5 A$ & Excalibur_c52167_355 & Xgwm156 & $11.44,6.39 / 7.34$ \\
\hline & $Q G W T-6 A, Q G W-6 A$ & $E_{2}, E_{4}$ & $6 \mathrm{~A}$ & wsnp_Ex_rep_c68165_6 & BS00034886_51 & $7.53,6.91$ \\
\hline & $Q G W T-7 B, Q G W-7 B$ & $\mathrm{E}_{4}, \mathrm{E}_{1} / \mathrm{E}_{4}$ & $7 \mathrm{~B}$ & $B A R C 014$ & BobWhite_c12256_96 & $4.22,12.43 / 15.57$ \\
\hline $\mathrm{GL}, \mathrm{GW}$ & $Q G L-5 B .1, Q G W-5 B .2$ & $\mathrm{E}_{1}, \mathrm{E}_{1}$ & $5 \mathrm{~B}$ & Xmag1242 & BobWhite_c28958_178 & $12.57,9.37$ \\
\hline \multicolumn{7}{|c|}{ a TGW, GL and GW mean thousand grain weight, grain length and grain width respectively. } \\
\hline \multicolumn{7}{|c|}{${ }^{\mathrm{b}} \mathrm{QTLs}$ detected on the same loci. } \\
\hline \multicolumn{7}{|c|}{${ }^{\mathrm{c}}$ Environments in which the QTLs were detected. } \\
\hline
\end{tabular}


Table 4

comparison of results with previous studies

\begin{tabular}{|c|c|c|c|c|}
\hline \multirow[t]{2}{*}{ Chromosome } & \multirow[t]{2}{*}{ Markers } & \multirow[t]{2}{*}{ QTL in this study } & \multicolumn{2}{|c|}{ QTL detected in previous studies } \\
\hline & & & Related treats ${ }^{a}$ & Reference \\
\hline \multirow[t]{6}{*}{$2 \mathrm{~B}$} & \multirow[t]{6}{*}{ Xgwm148 } & \multirow[t]{6}{*}{ QGWT-2B.1, QGW-2B.1 } & TGW & Luo et al. (2016) \\
\hline & & & TGW, GL, GW & Kumari et al. (2018) \\
\hline & & & TGW & Quarrie et al. (2005) \\
\hline & & & GW & Manickavelu et al. (2011) \\
\hline & & & TGW & Ramya et al. (2010) \\
\hline & & & TGW, GY & Groos et al. (2003) \\
\hline \multirow[t]{2}{*}{$4 \mathrm{~B}$} & BS00021984_51,IAAV971 & QGWT-4B.1, QGW-4B.1 & TGW & Chen et al. (2014) \\
\hline & \multicolumn{2}{|l|}{ wsnp_Ex_c3119_5763762 } & $\mathrm{GL}$ & Li (2017) \\
\hline $4 \mathrm{~B}$ & Xgwm251 & QGW-4B.3 & GW & Manickavelu et al. (2011) \\
\hline $5 \mathrm{~A}$ & Xgwm156 & QGWT-5A.3, QGW-5A.3 & $\mathrm{GL}$ & Manickavelu et al. (2011) \\
\hline \multirow[t]{2}{*}{$5 B$} & \multirow[t]{2}{*}{ Xgwm335 } & \multirow[t]{2}{*}{ QGL-5B.1, QGW-5B.2 } & TGW, GW & Ramya et al. (2010) \\
\hline & & & TGW & Cui et al. (2011) \\
\hline \multirow[t]{6}{*}{$5 B$} & \multirow[t]{6}{*}{ Xgwm499 } & \multirow[t]{6}{*}{ QGWT-5B, QGL-5B.2 } & TGW, GNS, SN & Li et al. (2015d) \\
\hline & & & TGW, GL, GW & Ramya et al. (2010) \\
\hline & & & TGW, GNS, GWS & Tang et al. (2011) \\
\hline & & & TGW & Krishnappa et al. (2017) \\
\hline & & & TGW & Li et al. (2012) \\
\hline & & & TGW & Quarrie et al. (2005) \\
\hline \multirow[t]{2}{*}{ 7B } & Xwmc517; & \multirow[t]{2}{*}{ QGW-7B, QGWT-7B } & \multirow[t]{2}{*}{ TGW } & Golabadi et al. (2010); \\
\hline & $X c f d 22$ & & & somers et al. (2004) \\
\hline
\end{tabular}

\section{Discussion}

Genetic relationship between 1000-grain weight and its main components

Grain length and grain width are the main components of 1000-grain weight, which are closely related to each other. Studies by Ramya (2010) and Gegas (2010) showed that the 1000-grain weight positive correlated with the grain length and grain width, and the correlation between 1000-grain weight and grain width was higher than that between 1000-grain weight and grain length. In this study, the correlation coefficient between 1000-grain weight and grain width, 1000 -grain weight and grain length, as well as grain length and grain width were $0.75,0.46$, and 0.29 , respectively. The correlation between 1000 -grain weight and grain width was much larger than that between 1000-grain weight and grain length. Similar results are detected in this study. The phenomenon that QTLs control associated traits tends to be distributed in the same or similar chromosome intervals, which was known as that one gene had multiple effects or tight linkage (Liang et al. 2010). In this study, fifteen Loci with multiple effects were detected, involving 37 QTLs that accounted for $90.2 \%$ of total numbers of QTLs detected. of which one locus simultaneously regulated 1000-grain weight, grain length and grain width, 11 loci simultaneously regulated 1000-grain weight and grain width, and 2 loci simultaneously regulated 1000-grain weight and grain length, one locus controlled both grain length and grain width. The positive additive effect values of different traits ' QTLs at the same loci were all derived from the same parent, which revealed the positive correlation at molecular level among 1000-grain weight, grain length and grain width.

Comparison of the present study with previous studies

By comparing the results of this study and previous studies, it was found that only nine alleles concerned with 1000-grain weight, grain length or grain width were in the same marker range as previously reported, while the others could not be identified for lack of a common molecular marker. Besides, the poor comparability of QTL mapping among different results may result from population size, genetic background, molecular marker types and so on.

Kumari et al. (2018) detected a locus for 1000-grain weight, grain length and grain width in the region Xgwm429-Xgwm148 on 2B chromosome. Luo et al. (2016), Quarrie et al. (2005), and Groos et al. (2005) have also detected QTLs for 1000-grain weight near the mark Xgwm148. Ramya et al. (2010) detected a QTL for 1000-grain weight near the marker Xbarc55 on 2B chromosome, which was stably expressed in multiple environments. By comparison in the highdensity genetic maps of micro-satellite markers (Somers et al. 2004), it was found that the distance between Xbarc55 and Xgwm148 was only 8 cM. QGWT2B.1 and QGW-2B.1 detected in this study located in the chromosome region Wsnp_EX_c1962_369626-XgWm148, and had the same molecular marker 
Xgwm148 as above studies. It was further confirmed that there was a locus for 1000-grain weight and related traits on chromosome 2B, which was closely linked to the molecular marker Xgwm148.

Chen et al. (2014) detected three main-effect QTLs for 1000-grain weight on chromosome 4B, of which, QGW4B-5 were found to be located in the same marker interval with QGWT-4B.1 and QGW-4B.1 detected in this study. There were three same tight-linked markers BS00021984, Tdurum and IAV971. Li (2017) also detected a QTL for grain length (QKI-WA-4B.1a) in this chromosome region, and this QTL had the same linked marker Wsnp_EX_c3119_5763762 as QGWT-4B.1 and $Q G W-4 B$. 1. At a distance of about $10 \mathrm{cM}$ from this locus, a locus simultaneously controlling 1000-grain weight and grain width, namely $Q G W T-4 B .2$ and QGW-4B.2, was also detected in this study. Chen et al. (2014) also detected a main-effect QTL (QGW4B-17) for 1000-grain weight Within a similar chromosomal region, therefore, these two Adjacent Chromosome region on chromosome 4B may also be important for the expression of grain-related traits, and more in-depth research is needed.

Ramya et al. (2010) detected a loci simultaneously controlled 1000-grain weight, grain length and grain width in the region Xbarc74-Xgwm499 of 5B chromosome. Li et al. (2015d), Krishnappa et al. (2017), Li et al. (2012), and Quarrie et al. (2005) detected one, and Tang et al. (2011) Detected Two QTLs for 1000-grain weight, linked with Molecular Marker Xgwm499. in this study, QGWT-5B and QGL-5B.2 were located nearby of Xgwm499 on chromosome 5B, and QGWT-5B is a major QTL stably expressed in 4 environments.

Golabadi et al. (2010) identified a main-effect QTL for 1000-grain weight on the 7B chromosome stably expressed under drought stress and normal water condition, which was closely linked with $X c f d 22-7 B$, the genetic distance between $X c f d 22-7 B$ and $X w m c 517$ was $13 c M$ by comparison in the high-density genetic maps of micro-satellite markers (Somers et al. 2004). In this study, the main-effect QTLs for GWT, GW and GL were also detected within a marker interval of about $10 \mathrm{cM}$ genetic distance from the Xwmc517. Whether these QTLs were the same as above loci should be further verified.

QTL with stable expression in multiple environments

The ultimate goal of QTL mapping is to detect stable and reliable quantitative trait loci and to closely link molecular markers for molecular marker assisted selection (MAS). 1000-grain weight, grain length and grain width are all typical quantitative traits, which are greatly affected by environment (Garcia-Suarez et al. 2010). QTLs expressed only in specific environment tend to be less effective, less stable and less reliable, while QTLs detected in multiple environments tend to have higher effect values and reliable. Two of the 51 QTLs for 1000-grain weight and related traits detected in this study were detected in four environments, six were detected in three environments, and 14 were detected in two environments. Total of 22 QTLs were detected in two or more environments, accounting for $43 \%$ of the number of QTLs detected in this study. It is necessary to deeply analyze and precisely locate the stable QTLs for Gene Cloning and marker-assisted selection.

\section{Declarations Conflict of interest}

Authors declare that they have no conflict of interest.

\section{Acknowledgments}

This work was financially supported by the National Key Research and Development Program of China (2016YFD0102004).

\section{References}

1. Alagu M, Kanako K, Hisako I, Michiko M, Yasunari O (2011) Molecular mapping of quantitative trait loci for domestication traits and $\beta$-glucan content in a wheat recombinant inbred line population. Euphytica 177:179-190. https://doi.org/10.1007/s10681-010-0217-9

2. Chen JS, Chen GF, LI QF, Zhang H, Shi CL, Sun CL, Deng ZY, Liu K, Gu ZQ, Tian JC (2014) Construction of Genetic Map Using Genotyping Chips and QTL Analysis of Grain Weight. Scientia Agricultura Sinica 47:4769-4779

3. Cui F, Ding A, Li J, Zhao CH, Li XF, Feng D, Wang XQ, Wang L, Gao JR, Wang HG (2011) Wheat kernel dimensions: how do they contribute to kernel weight at an individual QTL level? J Genet 90:409-425. https://doi.org/10.1007/s12041-011-0103-9

4. Garcia-Suarez JV, Roder MS, de Leon J (2010) Identification of QTLs and associated molecular markers of agronomic traits in heat (Triticum aestivum L.) under two conditions of nitrogen fertilization. Cereal Res Commun38:459 - 470

5. Gegas VC, Nazari A, Griffiths S, Simmonds J, Fish L, Orford S (2010) A genetic framework for grain size and shape variation in wheat. Plant Cell 22:10461056

6. Golabadi M, Arzani A, Mirmohammadi-Maibody SAM, Sayed-Tabatabaei BE, Mohammadi SA (2010) Identification of microsatellite markers linked with yield components under drought stress at terminal growth stages in durum wheat. Euphytica 177: 207-221. https://doi.org/10.1007/s10681-010-0242-8

7. Groos C, Robert N, Bervas E, Charmet G (2003) Genetic analysis of grain protein-content, grain yield and thousand-kernel weight in bread wheat. Theor Appl Genet 106:1032-1040. https://doi.org/10.1007/s00122-002-1111-1

8. Krishnappa G, Singh AM, Chaudhary S, Ahlawat AK, Singh SK, Shukla RB, Jaiswal JP, Singh GP, Solanki IS (2017) Molecular mapping of the grain iron and zinc concentration, protein content and thousand kernel weight in wheat (Triticum aestivum L.). PLoS ONE 12: e0174972

9. Kumari S, Jaiswal V, Mishra VK, Paliwal R, Balyan HS, Gupta PK (2018) QTL mapping for some grain traits in bread wheat (Triticum aestivum L.). Physiol Mol Biol Pla 24:909-920. https://doi.org/10.1007/s12298-018-0552-1

Page 9/11 
10. Liang Y, Zhang KP, Zhao L, Liu BQ, Meng Q, Tian J, Zhao S (2010) Identification of chromosome regions conferring dry matter accumulation and photosynthesis in wheat (Triticum aestivum L.) Euphytica 171:145- 156. https://doi.org/10.1007/s10681-009-0024-3

11. Li DD (2017) QTL Analysis of Main Agronomic and Yield Traits in “Weimai 8/Annong 91168” RIL population using SNP Genotyping Technology. Dissertation, Shandong Agricultural University

12. Li XM, Xia XC, Xiao YG, He ZH, Wang DS, Trethowan R, Wang HJ, Chen XM (2015d) QTL mapping for plant height and yield components in common wheat under water-limited and full irrigation environments. Crop Pasture Sci 66:660-670

13. Li SP, Wang CS, Chang XP, Jing RL (2012) Genetic dissection of developmental behavior of grain weight in wheat under diverse temperature and water regimes. Genetica 140:393-405. https://doi.org/10.1007/s10709-012-9688-Z

14. Luo W, Ma J, Zhou XH, Sun M, Kong XC, Wei YM, Jiang YF, Qi PF, Jiang QT, Liu YX, Peng YY, Chen GY, Zheng YL, Liu CJ, Lan XJ (2016) Identification of quantitative trait loci controlling agronomic traits indicates breeding potential of Tibetan semiwild wheat (Triticum aestivum ssp. tibetanum). Crop Sci 56:2410-2420

15. McIntosh RA, Devos KM, Dubcovsky J, Rogers WJ, Morris C F, Appels R, Anderson OD (2007) Catalogue of gene symbols for wheat: 2007 supplement. Yokohama, Japan, pp10-21

16. Quarrie SA, Steed A, Calestani C, Semikhodskii A, Lebreton C, Chinoy C, Steele N, Pljevljakusić D, Waterman E, Weyen J, Schondelmaier J, Habash DZ, Farmer P, Saker L, Clarkson DT, Abugalieva A, Yessimbekova M, Turuspekov Y, Abugalieva S, Tuberosa R, Sanguineti MC, Hollington PA, Aragués R, Royo A, Dodig D (2005) A high-density genetic map of hexaploid wheat (Triticum aestivum L.) from the cross Chinese Spring $\times$ SQ1 and its use to compare QTLs for grain yield across a range of environments. Theor Appl Genet 110: 865-880. https://doi.org/10.1007/s00122-004-1902-7

17. Ramya P, Chaubal A, Kulkarni K, Gupta L, Kadoo N, Dhaliwal HS, Chhuneja P, Lagu M, Gupta V (2010) QTL mapping of 1000-kernel weight, kernel length, and kernel width in bread wheat. J Appl Genet 51: 421-429. https://doi.org/10.1007/BF03208872

18. Tang YL, Li J, Wu YQ, Wei HT, Li CS, Yang WY, Chen F (2011) Identification of QTLs for yield-related traits in the ecombinant inbred line population derived from the cross between a synthetic hexaploid wheat-derived variety Chuanmai 42 and a Chinese elite variety Chuannong 16. Agr Sci China 10:1665-1680

19. Voorrips RE (2002) Map Chart: Software for the graphical presentation of linkage maps and QTL. J Hered 93:77-78

20. Yu ML, Zhao LM, Guo HJ, Gu JY, Li JH, Xie YD, Zhao SR, Liu LX (2014) QTL Mapping for kernel traits in wheat. Journal of Triticeae Crops 34:1029-1035

21. Zhang Hong (2013) QTL Mapping of seedling traits related to drought and low-phosphorus tolerance and construction of genetic linkage map in wheat. Dissertation, Shandong Agricultural University

\section{Figures}



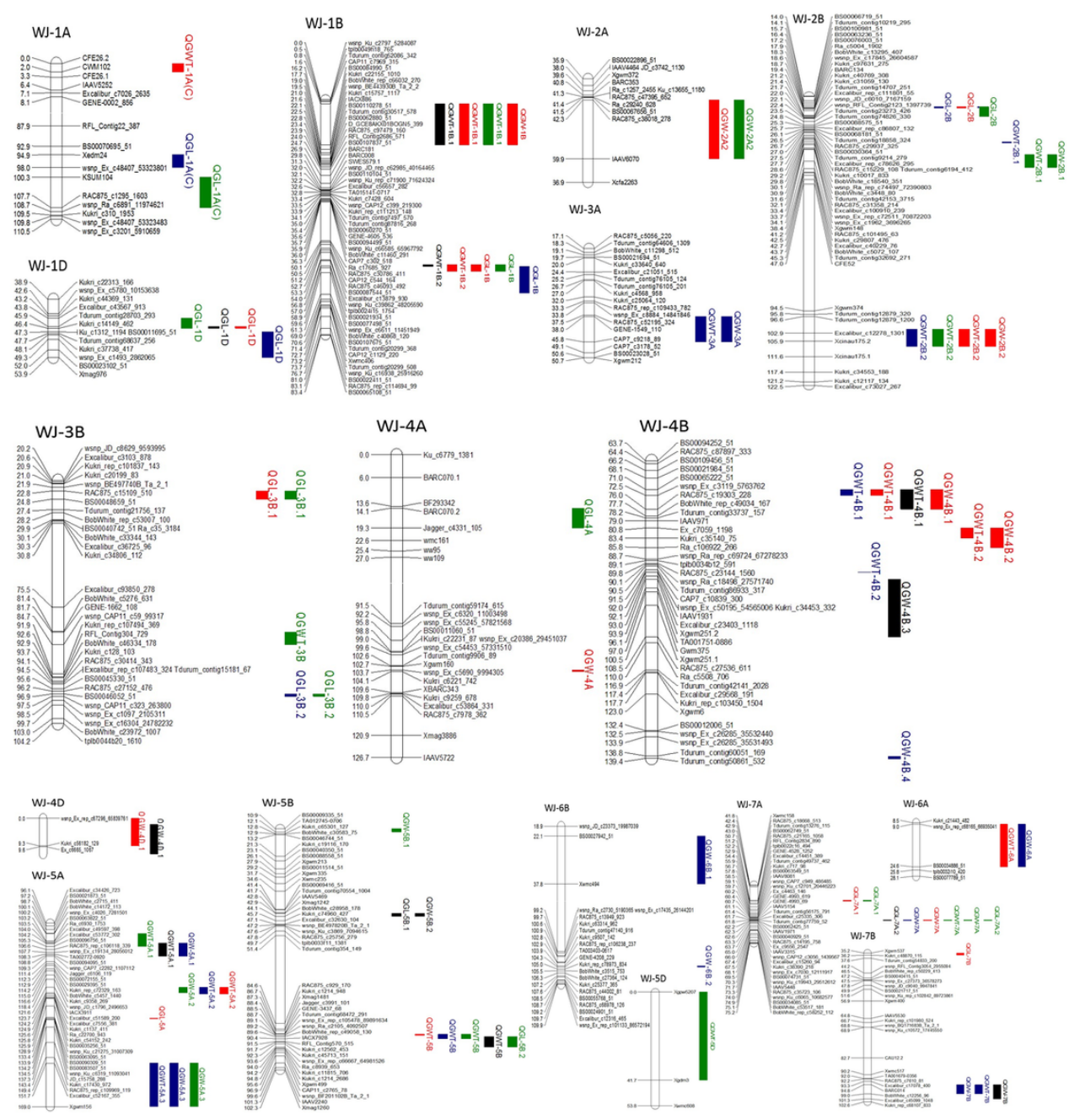

\section{Figure 1}

The position on the chromosomes of QTL concerned with 1000-grain weight and its main components in wheat. QTLs were indicated on the right side of each chromosome. Black, red, green and blue represent QTLs detected in E1, E2, E3 and E4 environments, respectively 